\title{
SINGLE-CENTER EXPERIENCE WITH THE THORATEC VENTRICULAR ASSIST DEVICE
}

R. Körfer, MD

A. El-Banayosy, MD

L. Arusoglu, MD

K. Minami, $\mathrm{MD}^{\mathrm{a}}$

M. M. Körner, MD

L. Kizner, $\mathrm{MD}^{\mathrm{a}}$

O. Fey, $\mathrm{RN}^{\mathrm{a}}$

U. Schütt, MD

M. Morshuis, $\mathrm{MD}^{\mathrm{a}}$

H. Posival, $\mathrm{MD}^{\mathrm{b}}$
Objective: The Thoratec ventricular assist device (Thoratec Laboratories, Pleasanton, Calif) is widely accepted for univentricular and biventricular support in patients with various indications. The aim of this study is to describe our experience with implantation of the Thoratec ventricular assist device in more than 100 patients. Methods: From March 1992 to June 1998, 114 patients (98 men and 16 women; mean age, 47.9 years) received the Thoratec ventricular assist device for a mean duration of 44.9 days. The patients were divided into 3 groups. Group 1 included 84 patients in whom the system was applied as a bridge-to-transplant procedure. Group 2 included 17 patients with postcardiotomy cardiogenic shock, and group 3 included 13 patients with cardiogenic shock of other causes. Results: Sixty-eight percent of patients in group 1 survived to transplantation with a posttransplant survival of $88 \%$. The only independent risk factor affecting survival was age more than 60 years. Survivals in groups 2 and 3 were $47 \%$ and 31\%, respectively. Main complications in all groups were bleeding, multiple organ failure, liver failure, sepsis, and neurologic disorders. Conclusions: The Thoratec ventricular assist device has proved to be a reliable device for bridge to transplantation and postcardiotomy support. Further studies are required on patient selection and on patient and device management to reduce the incidence of complications in these patient populations. (J Thorac Cardiovasc Surg 2000;119:596-600)
1 $n$ recent years, several devices for mechanical circulatory support have been developed to a stage at which they are no longer investigational devices. One of these systems is the Thoratec ventricular assist device (Thoratec VAD; Thoratec Laboratories Corp, Pleasanton, Calif). The system can be used for univentricular and biventricular support. Its main components are the blood pump (located paracorporeally), cannulas, and a hospital-based drive console, which is large but enables the patients to be fully ambulant on the system.

From the Department of Thoracic and Cardiovascular Surgery, Heart Center North Rhine-Westphalia, Ruhr University of Bochum, Bad Oeynhausen, ${ }^{\text {a }}$ Heart Surgery Clinic Karlsruhe. ${ }^{\text {b }}$

Supported by the German Association of Organ Recipients (Registered Association).

Received for publication Jan 21, 1999; revisions requested April 13, 1999; revisions received Oct 6, 1999; accepted for publication Oct 7, 1999.

Address for reprints: A. El-Banayosy, MD, Klinik für Thorax- und Kardiovaskularchirurgie, Herzzentrum NRW, Ruhr-Universität Bochum, Georgstr. 11, 32545 Bad Oeynhausen, Germany (Email: abanayosy@hdz-nrw.ruhr-uni-bochum.de).

Copyright @ 2000 by Mosby, Inc.

0022-5223/2000 $\$ 12.00+0 \quad \mathbf{1 2 / 1 / 1 0 3 5 1 9}$

doi: $10.1067 / \mathrm{mtc} .2000 .103519$
In addition, a portable VAD control unit (TLC-II) has recently become available for clinical evaluation at our center but is not included in the present study. The console version of the Thoratec VAD has been described in detail by Farrar and Hill. ${ }^{1}$ The system has been accepted for patients with postcardiotomy cardiogenic shock, ${ }^{2,3}$ as well as for bridge-to-transplant procedures, ${ }^{3-5}$ for durations as long as 515 days.

The aim of our work is to describe our 6-year experience with the implantation of the Thoratec VAD in 114 patients.

\section{Patients and methods}

Since our mechanical circulatory support program was started in September 1987, 382 patients (302 men and 80 women) ranging in age from 4 and 82 years (mean, $52 \pm 14$ years) received devices for mechanical circulatory assistance. Between March 1992 and June 1998, the Thoratec VAD was implanted in 114 patients (98 men and 16 women) between 11 and 64 years of age (mean, $47.9 \pm 14.4$ years). Nineteen patients were older than 60 years. Duration of support was 3 to 184 days (mean, $44.9 \pm 41.5$ days).

System selection. From March 1992 to March 1993, the Thoratec VAD was the only device available for bridging patients to transplantation at our center. Since the Novacor 
Table I. Outcome of patients bridged to transplantation with regard to preoperative risk factors for death

\begin{tabular}{lcc}
\hline Risk factor for death & Transplanted & $\begin{array}{c}\text { P value } \\
\text { (Cox regression) }\end{array}$ \\
\hline IABP $(\mathrm{n}=17)$ & $9(53 \%)$ & .9 \\
Reoperation $(\mathrm{n}=13)$ & $8(62 \%)$ & .8 \\
Acute renal failure $(\mathrm{n}=13)$ & $10(77 \%)$ & .5 \\
CPR $(\mathrm{n}=8)$ & $4(50 \%)$ & .3 \\
Age over 60 y $(\mathrm{n}=19)$ & $9(47 \%)$ & .05 \\
Liver insufficiency $(\mathrm{n}=40)$ & $28(70 \%)$ & .3 \\
BVAD $(\mathrm{n}=43)$ & $28(65 \%)$ & .7 \\
Previous VAD $(\mathrm{n}=6)$ & $2(33 \%)$ & .3 \\
Ventilation $(\mathrm{n}=15)$ & $7(47 \%)$ & .14 \\
\hline
\end{tabular}

$I A B P$, Intra-aortic balloon pumping; $C P R$, cardiopulmonary resuscitation.

and HeartMate left ventricular assist device (LVAD) systems have become additionally available in 1993 and 1994, respectively (Novacor: Baxter Healthcare Corp, Oakland, Calif; HeartMate: Thermo Cardiosystems Inc, Woburn, Mass), our system selection criteria have been modified. Patients are selected for biventricular support with the Thoratec VAD if one of the following conditions is present: central venous pressure greater than $20 \mathrm{~mm} \mathrm{Hg}$ and pulmonary artery pressure-central venous pressure gradient of less than $4 \mathrm{~mm} \mathrm{Hg}$, increased pulmonary vascular resistance $\left(>500\right.$ dynes $\cdot \mathrm{s}^{-1}$. $\mathrm{cm}^{-5}$ ), multiple organ dysfunction, or severe malignant arrhythmias refractory to medical therapy. The other patients receive a Thoratec LVAD or an implantable LVAD (Novacor or HeartMate provided the body surface area exceeds $1.5 \mathrm{~m}^{2}$ ).

Patients. The patients were divided into 3 groups as to indication of implantation.

Group 1 included 84 patients (72 men and 12 women; age range, 11-64 years; mean age, $46 \pm 15$ years) in whom the system was applied as a bridge-to-transplant procedure after having received maximum inotropic support and who were at imminent risk of death. Nineteen (23\%) of these patients were more than 60 years old. Seventeen $(20 \%)$ patients had been supported with an intra-aortic balloon pump, $13(16 \%)$ patients had undergone previous cardiac surgery, 13 (16\%) patients had acute renal failure (requiring renal replacement therapy preoperatively), and $8(10 \%)$ patients had cardiopulmonary resuscitation. Forty $(48 \%)$ patients had liver insufficiency (defined as bilirubin level $>2 \mathrm{mg} / \mathrm{dL}$, aspartate aminotransferase/alanine aminotransferase level of $3 \times$ normal value, $\gamma$-glutamyltransferase of $3 \times$ normal value, or alkaline phosphatase level of $1.5 \times$ normal value) before implantation of the device, and $15(18 \%)$ patients had received artificial ventilation. Statistical analyses of preoperative risk factors for death on support (Table I) were performed by using Cox regression analysis. Patients were censored at the time of device explant because of weaning or transplantation.

Duration of support in this group was 6 to 184 days with a mean duration of $50 \pm 44$ days. The underlying diagnoses were dilated cardiomyopathy in 53 patients, ischemic cardiomyopathy in 23 patients, end-stage valvular heart disease
Table II. Patient age and preoperative variables (mean values)

\begin{tabular}{lccc}
\hline & $\begin{array}{c}\text { LVAD } \\
(n=50)\end{array}$ & $\begin{array}{c}B V A D \\
(n=55)\end{array}$ & P value \\
\hline Age (y) & $49.9 \pm 12.0$ & $46.2 \pm 16.0$ & .17 \\
Hemodynamics & & & \\
$\quad$ CI $\left(\mathrm{L} \cdot \mathrm{min}^{-1} \cdot \mathrm{m}^{-2}\right)$ & $1.9 \pm 0.7$ & $2.2 \pm 0.2$ & .53 \\
$\mathrm{CVP}(\mathrm{mm} \mathrm{Hg})$ & $13.5 \pm 4.5$ & $17.6 \pm 4.0$ & .007 \\
PAP $(\mathrm{mm} \mathrm{Hg})$ & $33.3 \pm 9.0$ & $35.0 \pm 7.0$ & .54 \\
PVR & $260 \pm 135$ & $349 \pm 189$ & .29 \\
$\quad\left(\right.$ dynes $\left.\cdot \mathrm{s}^{-1} \cdot \mathrm{cm}^{-5}\right)$ & & & \\
SVR & $1510 \pm 891$ & $913 \pm 252$ & .06 \\
$\quad\left(\right.$ dynes $\left.\cdot \mathrm{s}^{-1} \cdot \mathrm{cm}^{-5}\right)$ & & & \\
Laboratory variables & & & .082 \\
AP $(\mathrm{U} / \mathrm{L})$ & $133.9 \pm 64.0$ & $174.6 \pm 85.0$ & .025 \\
BUN $(\mathrm{mg} / \mathrm{dL})$ & $80.2 \pm 39.3$ & $113.4 \pm 67.9$ & .024 \\
Bilirubin $(\mathrm{mg} / \mathrm{dL})$ & $1.8 \pm 0.9$ & $3.0 \pm 2.9$ & .377 \\
Creatinine $(\mathrm{mg} / \mathrm{dL})$ & $1.8 \pm 0.9$ & $2.1 \pm 1.3$ & .583 \\
$\gamma$-GT $(\mathrm{mg} / \mathrm{dL})$ & $53.9 \pm 44.1$ & $61.0 \pm 42.3$ & .619 \\
AST $(\mathrm{U} / \mathrm{L})$ & $126.2 \pm 213.4$ & $119.6 \pm 262.1$ & \\
ALT $(\mathrm{U} / \mathrm{L})$ & $143.7 \pm 180.6$ & $115.2 \pm 218.9$ & .607 \\
\hline
\end{tabular}

$C I$, Cardiac index; $C V P$, central venous pressure; $P A P$, pulmonary artery pressure; $P V R$, pulmonary vascular resistance; $S V R$, systemic vascular resistance; $A P$, alkaline phosphatase; $B U N$, blood urea nitrogen; $G T$, glutamyltransferase; $A L T$, alanine aminotransferase; $A S T$, aspartate aminotransferase.

in 4 patients, and miscellaneous other diagnoses in 4 patients. In 74 patients the Thoratec VAD was applied exclusively. Six additional patients had first received the centrifugal pump as femoro-femoral cardiopulmonary bypass, which was then replaced by Thoratec support, and 4 patients received the Thoratec device for right ventricular support in addition to the Novacor LVAS $(n=3)$ or the electrically driven TCI HeartMate device $(n=1)$. Forty-three patients received biventricular assistance, 34 patients received left ventricular assistance, 4 patients received Thoratec right ventricular assistance in combination with a LVAD, and 3 patients received Thoratec biventricular assist devices (BVADs) applied as a total artificial heart. For this purpose, the native heart was excised at atrioventricular level with the tricuspid and mitral valves left in place. Two straight long ventricular inflow cannulas were introduced into the right and left atria, respectively, and fixed with felt. The outflow cannulas were anastomosed to the aorta and pulmonary artery, respectively, in an end-to-end fashion. Five of the 84 patients had an aortic valve prosthesis, which was replaced by a bioprosthesis (n $=2$ ) or by the Thoratec VAD used as a total artificial heart (n $=3$ ).

Group 2 comprised 17 patients (15 men and 2 women; age range, $42-63$ years; mean age, $54 \pm 6$ years) who had postcardiotomy cardiogenic shock after coronary artery bypass grafting $(\mathrm{CABG}, \mathrm{n}=13)$, aortic valve replacement $(\mathrm{n}=2)$, tricuspid valve replacement $(\mathrm{n}=1)$, and concomitant mitral valve replacement and CABG $(n=1)$. Nine patients received the device in the operation after unsuccessful weaning from extracorporeal circulation, and 8 patients had cardiogenic 
Table III. Outcome

\begin{tabular}{lcccc}
\hline Outcome & $\begin{array}{c}\text { Bridging } \\
(n=84)\end{array}$ & $\begin{array}{c}\text { Postcardiotomy heart failure } \\
(n=17)\end{array}$ & $\begin{array}{c}\text { Miscellaneous } \\
(n=13)\end{array}$ & $\begin{array}{c}\text { Total } \\
(n=114)\end{array}$ \\
\hline Weaned & 2 & 1 & - & 3 \\
Transplanted & $57(68 \%)$ & $7(41 \%)$ & $4(31 \%)$ & $68(60 \%)$ \\
Discharged & $50(60 \%)$ & $8(47 \%)$ & $4(31 \%)$ & $62(54 \%)$ \\
Death on support & $25(30 \%)$ & $9(53 \%)$ & $9(69 \%)$ & $43(38 \%)$ \\
\hline
\end{tabular}

Table IV. Complications

\begin{tabular}{lccc}
\hline Complication & $B T T(n=84)$ & $P C(n=17)$ & Miscellaneous $(n=13)$ \\
\hline Bleeding & $26(31 \%)$ & $2(12 \%)$ & $2(15 \%)$ \\
Multiple organ failure & $20(24 \%)$ & $5(29 \%)$ & $4(31 \%)$ \\
Sepsis & $13(16 \%)$ & $6(35 \%)$ & $5(39 \%)$ \\
Liver failure & $18(21 \%)$ & $2(12 \%)$ & $1(8 \%)$ \\
Neurologic & $16(19 \%)$ & $3(18 \%)$ & $1(8 \%)$ \\
Pneumonia & $9(11 \%)$ & $2(12 \%)$ & $1(8 \%)$ \\
Gastrointestinal & $11(13 \%)$ & - & - \\
Exit site infection & $11(13 \%)$ & $2(12 \%)$ & - \\
Acute renal failure & $8(10 \%)$ & $1(9 \%)$ & - \\
Right heart failure & $4(12 \%)$ & $2(12 \%)$ & - \\
Hemolysis & $4(5 \%)$ & $1(6 \%)$ & - \\
Technical & $3(4 \%)$ & - & - \\
Mediastinitis & 1 & $(8 \%)$ & -
\end{tabular}

$B T T$, Bridge to transplant; $P C$, postcardiotomy cardiogenic shock.

"In patients with Thoratec left ventricular support (bridging, $n=34$; postcardiotomy, $n=11$ ).

shock postoperatively in the intensive care unit. In contrast to group 1, these patients were not listed for cardiac transplantation at the time of device implantation. Duration of support was 3 to 117 days (mean, $32 \pm 30$ days). Eleven patients received exclusive Thoratec support, 4 patients were first supported with the centrifugal pump (in 1 patient as femorofemoral cardiopulmonary bypass and in 3 patients as LVAD), and 2 were initially supported with the Abiomed biventricular system (Abiomed, Inc, Danvers, Mass), which was later replaced by a Thoratec BVAD. Six patients required biventricular assistance, and 11 patients left ventricular assistance.

The 13 patients of group 3 (11 men and 2 women; age range, $15-63$ years; mean age, $49 \pm 14$ years) received the Thoratec device for cardiogenic shock of different causes, such as acute myocardial infarction $(n=5)$, fulminant myocarditis $(n=2)$, primary graft failure $(n=1)$, and acute rejection $(n=5)$. At the time of implantation, these patients were not candidates for a transplant procedure. Duration of support in this group was 6 to 111 days, with a mean duration of $26 \pm 28$ days. Exclusive assistance with a Thoratec device was applied in 8 patients, and 5 patients had first received a centrifugal pump as a femoro-femoral cardiopulmonary bypass. Six patients required biventricular support, 5 patients required left ventricular support, and 2 patients had Thoratec BVADs applied as total artificial heart.

Values for preoperative variables in patients with left and biventricular assistance are summarized in Table II.

Cannulation approach and implantation criteria. In patients undergoing bridge-to-cardiac transplantation, ventricular cannulation is our method of choice because it provides higher VAD flow rate and better washing of the native left ventricle. In patients with postcardiotomy cardiogenic shock, atrial cannulation is preferred if a recovery of the ventricle can be expected. Our criteria for implantation have been published elsewhere. ${ }^{2,3}$

Anticoagulation protocol. In the first 24 hours postoperatively, the patients receive no anticoagulation. Thereafter, therapy is started with heparin according to the activated clotting time (1.5× initial value). After removal of chest drains, phenprocoumon (Narcumar) administration is started (dosage according to the international normalized ratio, 2.5-3.5). Aprotinin was administered preoperatively in patients undergoing reoperation and postoperatively in all patients with a bleeding complication.

Antibiotic protocol. Our antibiotic and infection management protocol consists of a short-term prophylactic administration of cefazolin ( $3 \times 2 \mathrm{~g}$ daily) in all patients until all drainages are removed. Percutaneous cannula exit sites are cleaned and prepared every 3 days with $2 \%$ merbromin. Patients with local exit site infections only do not receive specific systemic treatment. If systemic signs of infection develop, they are given antibiotics according to the antimicrobial sensitivity test (usually Staphylococcus aureus) starting with floxacillin (INN: flucloxacillin), 6 to $8 \mathrm{~g}$ daily. If methicillinresistant Staphylococcus aureus is present, therapy is started with vancomycin, with the dosage depending on blood level 
(20-40 mg/L). In case the infection cannot be controlled by this antibiotic regimen, rifampin (INN: rifampicin; $10 \mathrm{mg} / \mathrm{kg}$ body weight daily) according to liver and renal function is administered additionally. This additional antibiotic regimen is applied for at least 4 weeks.

\section{Results}

In group 1, 57 (68\%) patients underwent transplantation, $50(60 \%)$ of whom could be discharged from the hospital (Table III). Posttransplant survival through hospital discharge was $88 \%$. Two patients could be weaned from right ventricular support with the Thoratec device and were then successfully bridged to cardiac transplantation by using Novacor left ventricular assistance. Of those 3 patients who had received the device as a total artificial heart, 2 could be discharged after transplantation, and the third patient died from multiple organ failure and sepsis under support. Both patients with a bioprosthesis in the aortic position were survivors; one had a transient ischemic attack after implantation. The only independent risk factor for death on the assist device turned out to be patient age over 60 years (Table I). Main complications were bleeding $(31 \%)$, multiple organ failure $(24 \%)$, liver failure $(21 \%)$, and neurologic disorders $(18 \%)$, which occurred from postoperative days 5 to 120 (mean, $37 \pm$ 26 days; Table IV). Six of 11 patients with gastrointestinal complications had to undergo surgery under support, with 5 survivors and 1 death.

In group 2, 1 patient who had undergone CABG could be weaned and discharged from the hospital. Seven (41\%) patients whose native heart did not recover had successful transplantation and were discharged (posttransplant survival rate, 100\%). Survival in this group was $47 \%$ (Table III). Five (36\%) of 14 patients after CABG, both of whom had aortic valve replacement, and the patient with the combined procedure were survivors. Six (55\%) of 11 patients with LVAD and 2 (33\%) of 6 patients with BVAD support survived to be discharged. Most frequent complications were sepsis $(35 \%)$ and multiple organ failure (29\%, Table IV).

In group 3, only $4(31 \%)$ of the 13 patients ( 1 with myocarditis, 1 with rejection, 1 with acute myocardial infarction, and 1 with primary graft failure) underwent transplantation and were discharged from the hospital (posttransplant survival, 100\%; Table III). Both patients with the total artificial heart, 1 (20\%) of 5 patients with an LVAD, and 1 (17\%) of 6 patients with a BVAD survived. Main complications were sepsis (39\%) and multiple organ failure (31\%, Table IV).

Altogether, 68 (60\%) patients underwent heart transplantation, and 6 of them died after the procedure.
Sixty-two (54\%) patients could be discharged home. The posttransplant survival was $91 \%$.

In 5 patients ( 3 bridge-to-transplant and 2 postcardiotomy patients) the sternum could not be completely closed immediately at the end of surgery but had to be closed 2 to 5 days postoperatively. Two of these patients (1 bridge-to-transplant and 1 postcardiotomy patient) died from multiple organ failure, and the other 3 patients underwent successful transplantation.

Right heart failure developed in 5 patients initially supported with an LVAD. In two cases, it was refractory to pharmacologic therapy, which did not include nitric oxide because it is not available in our operating room. These two patients received the Thoratec right ventricular assist device in addition to the primary left ventricular support. One patient supported as a bridge-to-transplant procedure died OF multiple organ failure, and the other patient supported for postcardiotomy cardiogenic shock was discharged after successful transplantation.

The main causes of death in all groups $(n=52)$ were multiple organ failure and sepsis.

During our cumulative experience of 13.2 patientyears, there were no major technical problems (eg, rupture of blood sac and VAD replacement). Minor technical complications occurred in 4 patients (LVAD compressor failure and driveline kinking), but these did not have any negative effect for the patients.

\section{Discussion}

This study describes our experience with the Thoratec VAD in more than 100 patients with different indications. Generally, our cumulative experience of 13.5 patient-years without major technical problems has proved the reliability of the system. Another advantage of the device is its versatility because it can be applied for right, left, and biventricular support with the possibility of atrial and ventricular cannulation, as has also been described by other authors. ${ }^{1,4-8}$ However, the size of the drive console and the paracorporeal location of the blood pumps make the system unsuitable in some patients for long-term support, although other centers have had successful experience up to 515 days. To address these issues, Thoratec has recently introduced a small portable briefcase-size control unit, which greatly improves patient mobility, ${ }^{6,7,9}$ and they are developing an intracorporeal version of their ventricular assist device.

Mechanical circulatory support still is associated with serious complications in these critically ill patient populations. Bleeding amounted to $26 \%$ in our collective experience, which is comparable with or lower than that found in other reports, ${ }^{1,8}$ although these inves- 
tigations only considered patients bridged to transplantation. Compared with our early experience, the bleeding complication declined as a result of the learning curve, ${ }^{3,10}$ principally because of our efforts to maintain meticulous hemostasis during surgical implantation. The high prevalence of multiple organ failure, liver failure, and sepsis resulted from the poor preoperative condition of the patients who often had multiple organ dysfunction and, with surgical trauma, frequently have organ failure.

The prevalence of all forms of neurologic complications $(\mathrm{n}=20,18 \%)$ was high but within the range for other devices; for example, in the HeartMate Food and Drug Administration clinical trial, the prevalence of neurologic dysfunction was $21 \% .{ }^{11}$ Not all of the complications were considered to be related to devices. Three patients had cerebral bleeding, which occurred after cerebral infarction in one case, and in the other two cases probably resulted from anticoagulation. Four patients had transient ischemic attacks, and in one of them the attack was associated with an infection complication. Two patients, who had received Thoratec support during cardiopulmonary resuscitation, had paraplegia detected shortly after implantation. Eleven patients had hemiplegia during support 2 to 18 weeks postoperatively, 7 of whom recovered and underwent transplantation. In 4 patients, hemiplegia occurred after an infection. Thus $12(11 \%)$ patients (11 with hemiplegia and 1 with cerebral bleeding) had major neurologic disorders, which could be related to a device, and in the absence of infection, the prevalence was $7 \%$.

Gastrointestinal complications were upper gastrointestinal tract bleeding and cholecystitis in 3 patients each and mesenteric ischemia in 5 patients. Abdominal surgery was necessary under support in 6 patients (cholecystectomy and hemicolectomy in 2 cases each and appendectomy and splenectomy in 1 case each). The prevalence of exit site infections was considerably low because of our strict redressement regimen under sterile conditions by a trained VAD team. It did not at all occur in patients with postcardiotomy cardiogenic shock and with other indications, probably because of the lower duration of support.

The Thoratec VAD has proved to be a reliable device for bridge-to-transplant and postcardiotomy support. The large hospital-based console, however, represented a disadvantage limiting the mobility of the patient. Currently, the portable VAD driver has become available, which is significantly improving the quality of life of VAD patients. ${ }^{6,79}$ Patient age of more than 60 years turned out to be the only independent risk factor affecting survival.

\section{REFERENCES}

1. Farrar DJ, Hill JD. Univentricular and biventricular Thoratec VAD support as a bridge to transplantation. Ann Thorac Surg 1993;55:276-82.

2. Körfer R, El-Banayosy A, Posival H, Minami K, Kizner L, Arusoglu L, et al. Mechanical circulatory support with the Thoratec ventricular assist device in patients with postcardiotomy cardiogenic shock. Ann Thorac Surg 1996;61:314-6.

3. Körfer R, El-Banayosy A, Posival H, Minami K, Körner MM, Arusoglu L, et al. Mechanical circulatory support: the Bad Oeynhausen experience. Ann Thorac Surg 1995;59(Suppl):S56-62.

4. Farrar DJ, Hill JD, Pennington DG, McBride LR, Holman WL, Kormos RL, et al. Preoperative and postoperative comparison of patients with univentricular and biventricular support with the Thoratec ventricular assist device as a bridge to cardiac transplantation. J Thorac Cardiovasc Surg 1997;113:202-9.

5. Griffith BP, Kormos RL, Nastala CJ, Winowich S, Pristas JM. Results of extended bridge to transplantation: window into the future of permanent ventricular assist devices. Ann Thorac Surg 1996;61:396-8.

6. Farrar DJ, Buck KE, Coulter JH, Kupa EJ. Portable pneumatic biventricular driver for the Thoratec ventricular assist device. ASAIO J 1997;43:M631-4.

7. von Segesser LK, Tkebuchava T, Leskosek B, Marty B, Pei YC, Turina M. Biventricular assist using a portable driver in combination with implanted devices: preliminary exerpience. Artif Organs 1997;21:72-5.

8. Pennington DG, McBride LR, Peigh PS, Miller LW, Swartz MT. Eight years' experience with bridging to cardiac tranplantation. J Thorac Cardiovasc Surg 1994;107:472-81.

9. Farrar DJ, Körfer R, El-Banayosy A, Posival H, Loisance D, Tixier D. First clinical use of the Thoratec TLC-II portable VAD driver in ambulatory and patient discharge settings [abstract]. ASAIO J 1998;44:35A.

10. Körfer R, El-Banayosy A, Arusoglu L, Minami K, Breymann T, Seifert $\mathrm{D}$, et al. Temporary pulsatile ventricular assist devices and biventricular assist devices. Ann Thorac Surg 1999;68:678-83.

11. HeartMate Implantable Pneumatic Left Ventricular Assist System Directions for Use. Woburn [MA \}.ThermoCardiosystems, Inc; 1995. 